\title{
IMPROVEMENT OF BER PERFORMANCE IN MIMO-CDMA SYSTEMS BY USING INITIAL-PHASE OPTIMIZED GOLD CODES
}

\author{
Ibrahim Develi $^{*}$ - Meryem Filiz ${ }^{* *}$
}

\begin{abstract}
This paper describes a new approach to improve the bit error rate (BER) performance of a multiple-input multiple-output code-division multiple-access (MIMO-CDMA) system over quasi-static Rayleigh fading channels. The system considered employs robust space-time successive interference cancellation detectors and initial-phase optimized Gold codes for the improvement. The results clearly indicate that the use of initial-phase optimized Gold codes can significantly improve the BER performance of the system compared to the performance of a multiuser MIMO-CDMA system with conventional nonoptimized Gold codes. Furthermore, this performance improvement is achieved without any increase in system complexity.

K e y w ords: wireless communications, spread spectrum communications, digital communications, multiple access systems, MIMO-CDMA systems, BER performance improvement, initial-phase optimized Gold codes
\end{abstract}

\section{INTRODUCTION}

In recent years, wireless communication systems using multiple antennas at both the base station and mobile stations have been developed to enhance the capacity and increase the system performance [1-3]. By the integration of multiple-input multiple-output (MIMO) and code-division multiple-access (CDMA), the resultant MIMO-CDMA system can further improve the transmission rate over the traditional single-input single-output (SISO) based communication systems $[1,4,5]$.

Although a multiuser MIMO-CDMA communication system has much better performance compared with a single antenna CDMA system, time-delay mismatch between transmit-receive antenna pairs can result in significant performance degradation. Therefore, many extensive studies have been carried out in order to mitigate the negative effects of time-delay mismatch in multiuser detection (MUD) [6-11]. In [11], Zha and Blostein introduced a new robust multiuser signal detector for asynchronous CDMA uplink channels under delay mismatch. Besides, in that study, a robust implementation of successive interference cancellation (SIC) has also been proposed. It was shown by computer simulation that the proposed robust SIC detector outperforms the conventional decorrelating detector when delay estimation error is present. As an extension of the multistage robust SIC [11], a multiuser MIMO- CDMA environment was considered by Lu in [12]. A robust space-time SIC (RSTSIC) that combines the original robust SIC with maximal ratio combining (MRC) of the multiple data streams arriving at each mobile was proposed and its bit error rate (BER) performance was evaluated. The authors in their work have shown that the RSTSIC receiver under timing mismatch is able to achieve close performance to that of the space-time decorrelator (STD) with perfect timing estimates [12]. In all of the above papers, performance in terms of signal-to-noise ratio (SNR) versus BER is presented for non-optimized pseudo-noise $(\mathrm{PN})$ codes.

An important step in the design process of a CDMAbased system is the selection of a spreading code family and optimization of its sub-code set. In [13], the authors have presented results concerning the influence of the choice of various initial-phase optimization criteria for a spreading code set on the SNR performance of an asynchronous short-code direct sequence DSCDMA system. In the initial-phase optimization, the auto-optimal least sidelobe energy (AO/LSE), the least sidelobe energy auto-optimal (LSE/AO), the maximum sidelobe energy auto-optimal (MSE/AO), the cross-optimal minimum mean-square cross-correlation (CO/MSQCC) and minimum mean square cross-correlation cross-optimal (MSQCC/CO) criteria were considered [13,14]. It was also emphasized that caution should be exercised when choosing PN codes for the numerical analysis and simulation of a CDMA system. Therefore, many researchers have been inspired by the concept of the initial-phase optimization and used initial-phase optimized PN code sequences to analyze or test their CDMA based systems [15-20].

In this paper, the BER performance of a multiuser MIMO-CDMA system with initial-phase optimized Gold codes is evaluated by computer simulation. It is shown that the use of initial-phase optimized Gold codes can significantly improve the BER performance compared to the performance of a multiuser MIMO-CDMA system employing conventional non-optimized Gold codes [21]. To the best of our knowledge, there has been no attempt in the open literature to evaluate the BER performance

\footnotetext{
* Department of Electrical \& Electronics Engineering, Faculty of Engineering, Erciyes University, 38039, Kayseri, Turkey, develi@erciyes.edu.tr ${ }^{* *}$ Graduate School of Natural and Applied Sciences, Erciyes University, 38039, Kayseri, Turkey
} 


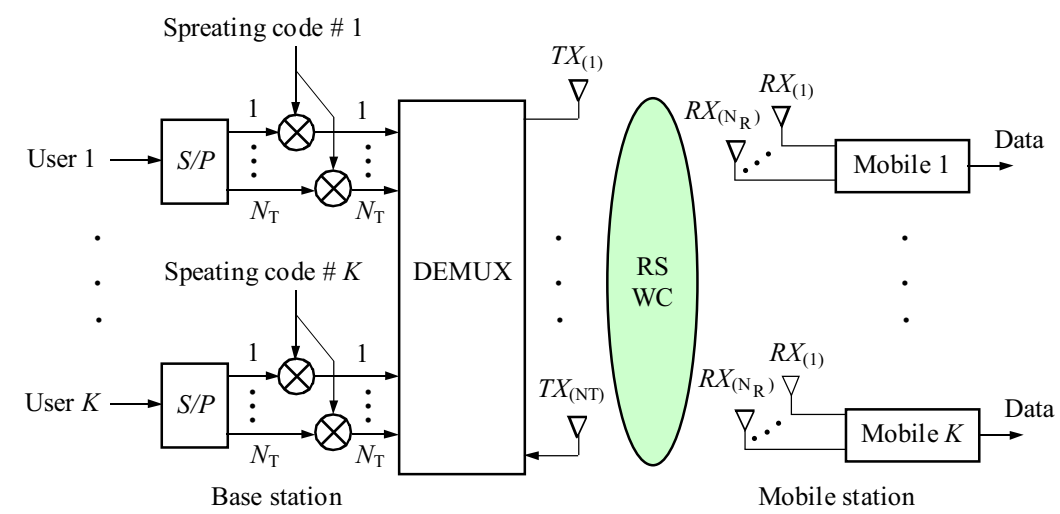

Fig. 1. Block diagram of a multiuser downlink MIMO-CDMA system, RSWC - stands for the rich scattering wireless channel

by exploiting the initial-phase optimized Gold codes in case of a multiuser MIMO-CDMA system that employs RSTSIC detectors.

The paper is organized as follows. Section 2 presents the system description presented in [12], while Section 3 summarizes the basic principles of the phase optimization methods introduced in the literature. Section 4 presents the computer simulation results on the BER performance of a multiuser MIMO-CDMA system employing various initial-phase optimized Gold codes. Finally, appropriate conclusions are offered in Section 5.

We conclude the introduction with a notational definition: Vectors and matrices are denoted by lower-case or upper-case bold letters. The operators $(\cdot)^{\top},(\cdot)^{H}$ and $(\cdot)^{*}$ denote transpose, conjugate transpose, and conjugate, respectively. $\mathbf{I}_{K M}$ is used to denote the $K \times M$ identity matrix, and $\otimes$ denotes the Kronecker product. For complex numbers and matrices, $\operatorname{Re}\{\cdot\}$ represents the real part while $\operatorname{abs}(\cdot)$ and $\operatorname{sign}(\cdot \cdots)$ denote absolute value and the sign, respectively. Lastly, we define the conventional 2-norm function with $\|\cdot\|$.

\section{SYSTEM DESCRIPTION}

\subsection{System model}

Downlink of a $K$-user MIMO-CDMA wireless communication system is considered, where the spreading codes of all users are assumed to be known at the receiver. Figure 1 shows the general block diagram of a multiuser downlink MIMO-CDMA system. As shown in Fig. 1, $N_{T}$ transmit antennas are located at the base station (BS) and $N_{R}$ receive antennas are located at each mobile station (MS). In the transmitter (BS), each user's symbols are demultiplexed into $N_{T}$ substreams, corresponding to the $N_{T}$ transmit antennas. The $K N_{T}$ data substreams are each spread into chips by an assigned spreading code of length $L$ and then transmitted through a rich-scattering environment.

The receiver (MS) employs an array of $N_{R}$ receive antennas to demodulate $N_{T}$ independent data substreams transmitted by the BS. We consider the case where the receive antennas are separated enough that the complex fading coefficients among the antennas are uncorrelated. We also assumed that the time delays between antenna pairs are independent, and are limited to occur within one symbol interval. The received baseband signal, $r_{p}(t)$, for a frame of $M$ data bits at the $p$-th antenna is given by

$$
\begin{array}{r}
r_{p}(t)=\sum_{m=1}^{M} \sum_{n=1}^{N_{T}} \sum_{k=1}^{K}\left(c_{n, p} a_{k, n} s_{k}\left(t-m T_{s}-\tau_{n, p}\right) b_{k, n}(m)\right. \\
\left.+n_{p}(t)\right)
\end{array}
$$

where $c_{n, p}$ is the channel coefficient corresponding to the fading channel between the $n$-th transmit antenna at the $\mathrm{BS}$ and the $p$-th receive antenna at the MS, $a_{k, n}$ is the amplitude of the $k$-th user's $n$-th substream, $s_{k}(t)$ is the spreading code of the $k$-th user, $T_{s}$ is the symbol interval, $\tau_{n, p}$ is the time delay of the path between the $n$-th transmit anntenna and the $p$-th receive antenna, $b_{k, n}(m)$ is the binary phase shift keying (BPSK) modulated data symbol of the $k$-th users $n$-th substream, $n_{p}(t)$ is the additive white Gaussian noise (AWGN) on receive antenna $p$, and $M$ is the frame size. The received discrete-time matched filter signal at the $p$-th receive antenna can be written as [12]

$$
\boldsymbol{r}_{p}=\mathbf{S}_{p} \widetilde{\mathbf{C}}_{p} \mathbf{A} \boldsymbol{b}+\mathbf{n}_{p}
$$

where

$$
\mathbf{S}_{p}=\left[\mathbf{S}_{k, n, p}(1) \mathbf{S}_{k, n, p}(2) \ldots \mathbf{S}_{k, n, p}(M)\right]
$$

is the spreading code matrix formed from concatenating the matrices

$$
\mathbf{S}_{k, n, p}=\left[\mathbf{s}_{1,1, p}(i) \ldots \mathbf{s}_{1, N_{T}, p}(i) \mathbf{s}_{2,1, p}(i) \ldots \mathbf{s}_{K, N_{T}, p}(i)\right]
$$

where $\mathbf{S}_{k, n, p}(i)$ is the $i$-th bit spreading code matrix. Matrix $\widetilde{\mathbf{C}}_{p}$ is block diagonal of size $K M N_{T} \times K M N_{T}$ 
defined by $K M$ instances of the channel matrix $\mathbf{C}_{p}$ along the main diagonal

$$
\widetilde{\mathbf{C}}_{p}=\mathbf{I}_{K M} \otimes \operatorname{diag}(\underbrace{\mathbf{C}_{p} \ldots \mathbf{C}_{p}}_{N_{T}}) .
$$

The matrix $\mathbf{C}_{p}$ in (5) is the $N_{T} \times N_{T}$ channel matrix given by

$$
\mathbf{C}_{p}=\operatorname{diag}\left(\begin{array}{llll}
c_{1, p} & c_{2, p} & \ldots & c_{N_{T}, p}
\end{array}\right)
$$

A in (2) is a $K M N_{T} \times M K N_{T}$ amplitude diagonal matrix, and $\boldsymbol{b}$ is $K M N_{T} \times 1$ binary data vector defined by

$$
\boldsymbol{b}=\left[\begin{array}{llll}
\boldsymbol{b}_{k, n}^{\top}(1) \boldsymbol{b}_{k, n}^{\top}(2) & \ldots & \boldsymbol{b}_{k, n}^{\top}(M)
\end{array}\right]^{\top}
$$

where $\boldsymbol{b}_{k, n}(i)=\left[b_{1,1}(i) \ldots b_{1, N_{T}}(i) b_{2,1}(i) \ldots b_{K, 1}(i) \ldots\right.$ $\left.b_{K, N_{T}}(i)\right]^{\top}$. Vector $\boldsymbol{n}_{p}$ is $(M+1) L \times 1$ zero-mean unit variance complex AWGN vector with independently and identically distributed (i.i.d.) components. It should be noted that all the sub-streams corresponding to a particular user have equal transmission energy, weighted by $1 / \sqrt{N_{T}}$ in order to restrict the total power output for the users. The channel is assumed to be quasi-static; ie $\widetilde{\mathbf{C}}_{p}$ $\left(p=1, \ldots, N_{R}\right)$ is fixed over the duration of the frame and is assumed to be perfectly estimated at the receiver $[12,22]$.

\subsection{Robust Space-Time SIC (RSTSIC)}

Let the estimated time delay from the $n$-th transmit antenna to the $p$-th receive antenna be $\hat{\tau}_{n, p}=\left(\rho_{n, p}+\right.$ $\left.\hat{\delta}_{n, p}\right) T_{c}$, where $T_{c}$ represents the chip interval, $\rho_{n, p} \in$ $\{0,1, \ldots, L-1\}$ and $\hat{\delta}_{n, p} \in[0,1)$ denotes the fractional part of the estimated time delay. Then, the $k$-th user's signature waveform for the $i$-th interval, $\mathbf{s}_{k, n, p}(i)$, can be defined as the weighted sum of two signals

$$
\mathbf{s}_{k, n, p}(i)=\hat{\mathbf{s}}_{k, n, p}(i)+\left(\delta_{n, p}-\hat{\delta}_{n, p}\right) \Delta \mathbf{s}_{k, n, p}(i)
$$

where $\hat{\boldsymbol{s}}_{k, n, p}(i)$ is the estimated code vector, $\delta_{n, p}$ is the fractional part of the true time delay, and $\Delta \boldsymbol{s}_{k, n, p}(i)$ is the error code vector. Based on (8), we can assume each user as the combination of two virtual users, one with $\hat{\mathbf{s}}_{k, n, p}(i)$ and the other with $\Delta \boldsymbol{s}_{k, n, p}(i)$. The basic steps for the implementation of the RSTSIC detector can be summarized as following

Step 1: Initialization: For $1 \leq k \leq K, 1 \leq m \leq M$, set: $\hat{a}_{k}^{0}(m)=0, \hat{b}_{k}^{0}(m)=0$ and $\Delta \hat{a}_{k}^{0}(m)=0$.

\section{Step 2: REPEAT}

Step 3: Determine user $k$ 's remaining data information for the $(j+1)$-th iteration and update the amplitude and data bits

$$
I_{k, n, p}^{j+1}(m)=\left\{\left(\hat{\boldsymbol{s}}_{k, n, p}(m)\right)^{H}\left(\mathbf{r}_{p}^{j+1}\right) /\left\|\hat{\mathbf{s}}_{k, n, p}(m)\right\|\right\},
$$

$$
\begin{gathered}
I_{k, n}^{j+1}(m)=\operatorname{Re}\left[\sum_{p=1}^{N_{R}} c_{n, p}^{*} I_{k, n, p}^{j+1}(m) / \sum_{p=1}^{N_{R}} c_{n, p}^{*} c_{n, p}\right], \\
\hat{a}_{k, n}^{j+1}(m)=\operatorname{abs}\left(I_{k, n}^{j+1}(m)+\hat{a}_{k, n}^{j}(m) \hat{b}_{k, n}^{j}(m)\right) \\
\hat{b}_{k, n}^{j+1}(m)=\operatorname{sign}\left(I_{k, n}^{j+1}(m)+\hat{a}_{k, n}^{j}(m) \hat{b}_{k, n}^{j}(m)\right)
\end{gathered}
$$

where $I_{k, n, p}^{j+1}(m)$ is the matched filter output at the $(j+$ $1)$-th iteration for the $m$-th bit of the $n$-th substream of the $k$-th user, $I_{k, n}^{j+1}(m)$ is the normalized output after MRC at the $(j+1)$-th iteration, $\mathbf{r}_{p}^{j+1}$ is the stripped composite received signal at the $(j+1)$-th iteration, $\hat{a}_{k, n}^{j}(m)$ is the amplitude information collected up to the $j$-th iteration, $\hat{a}_{k, n}^{j+1}(m)$ is the amplitude information collected up to the $(j+1)$-th iteration, $\hat{b}_{k, n}^{j}(m)$ is the data bit estimate at the $j$-th iteration for the $m$-th bit of $k$-th's user's $n$-th substream and $\hat{b}_{k, n}^{j+1}(m)$ is the data bit estimate at the $(j+1)$-th iteration for the $m$-th bit of $k$-th's user's $n$-th substream.

Step 4: Update the estimate of the remaining received signal

$$
\hat{\boldsymbol{r}}_{k, n, p}^{j+1}=\sum_{m=1}^{M} c_{n, p} I_{k, n}^{j+1}(m) \hat{\mathbf{s}}_{k, n, p}(m)
$$

where $\hat{\boldsymbol{r}}_{k, n, p}^{j+1}$ is the reconstructed received signal at antenna $p$ using only the extracted information from the $(j+1)$-th iteration for the $k$-th user's $n$-th substream.

Step 5: Estimate the residual signal of the $k$-th user due to timing error as

$$
\Delta \boldsymbol{r}_{k, n, p}^{j+1}=\boldsymbol{r}_{p}^{j}-\hat{\mathbf{r}}_{k, n, p}^{j+1}+\Delta \hat{\mathbf{a}}_{k, n, p}^{j} \mathbf{e}_{k, n, p}^{j}
$$

where $\mathbf{r}_{p}^{j}$ is the stripped composite received signal at the $j$-th iteration, $\Delta \hat{\mathbf{a}}_{k, n, p}^{j}$ is the amplitude of the error vector at the $j$-th iteration and $\boldsymbol{e}_{k, n, p}^{j}$ is the error vector at the $j$-th iteration.

Step 6: Update the amplitude of the error vector

$$
\boldsymbol{e}_{k, n, p}^{j+1}=\sum_{m=1}^{M} c_{n, p} \hat{b}_{k, n}^{j+1}(m) \Delta \hat{\mathbf{s}}_{k, n, p}(m)
$$

$\Delta \hat{\mathbf{a}}_{k, n, p}^{j+1}=$

$$
\operatorname{Re}\left[\left(\boldsymbol{e}_{k, n, p}^{j+1}\right)^{H}\left(\Delta \boldsymbol{r}_{p, n, p}^{j+1}\right) /\left(c_{n, p}^{*} c_{n, p} \boldsymbol{e}_{k, n, p}^{j+1}\left\|\boldsymbol{e}_{k, n, p}^{j+1}\right\|\right)\right]
$$

where $\mathbf{e}_{k, n, p}^{j+1}$ error vector at the $(j+1)$-th iteration, $\Delta \hat{\mathbf{a}}_{k, n, p}^{j+1}$ is the amplitude of the error vector at the $(j+1)$-th iteration.

Step 7: Strip away newly extracted information by subtracting the estimated remaining signal and the estimated error signal

$$
\boldsymbol{r}_{p}^{j+1}=\mathbf{r}_{p}^{j}-\boldsymbol{r}_{k, n, p}^{j+1}-c_{n, p} \Delta \hat{\mathbf{a}}_{k, n, p}^{j+1} \mathbf{e}_{k, n, p}^{j+1}
$$

where $\boldsymbol{r}_{p}^{j+1}$ is the stripped composite received signal at the $(j+1)$-th iteration.

Step 8: UNTIL $\left|\hat{a}_{k, n}^{j+1}(m)-\hat{a}_{k, n}^{j}(m)\right|<$ a specific threshold. 


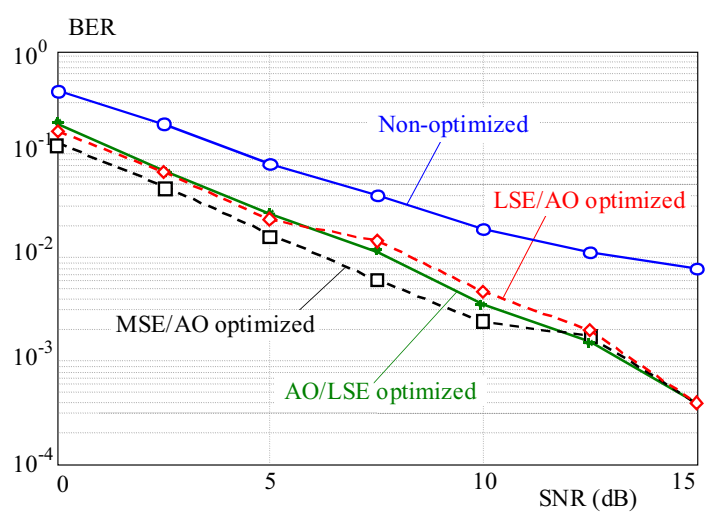

Fig. 2. BER performance of MIMO-CDMA system that used $\mathrm{LSE} / \mathrm{AO}, \mathrm{AO} / \mathrm{LSE}$ and MSE$/ \mathrm{AO}$ optimized Gold codes $(\mathrm{NFR}=0 \mathrm{~dB})$

\section{PRINCIPLES OF PHASE OPTIMIZATION METHODS}

Searching for a family or set of codes in which the maximum absolute values $\left(\theta_{A}, \theta_{C}\right)$ of the even (periodic) auto-correlation function (ACF) and the cross-correlation function (CCF) are small, is a general approach for the selection of CDMA codes. In addition to this requirement, it is also desired to find a subset in which the maximum values of the odd functions $\left(\hat{\theta}_{A}, \hat{\theta}_{C}\right)$ are small. The essential aim is to minimize the parameters $\hat{\theta}_{A}$ and $\hat{\theta}_{C}$ by finding the optimum phase-shift combination for each of the $K$ codes in a set. After the completion of this step, it is expected that the optimum phase shifts determined will maximize the SNR and minimize the bit error probability, $i e$; minimize the amount of multiple access interference (MAI) [13].

The average SNR for the $j$-th correlation receiver can be expressed as $[23,24]$

$$
S N R_{j}=\left\{\left(6 p^{3}\right)^{-1} \sum_{\substack{i=1 \\ i \neq j}}^{K} r_{i, j}+\frac{N_{0}}{2 E_{b}}\right\}^{-1 / 2}
$$

where $p$ is the code length (period) and $r_{i, j}$ is the average interference parameter (AIP) defined by

$$
\begin{aligned}
r_{i, j} & =\left\lfloor 2 \mu_{i, j}(0)+\mu_{i, j}(1)\right\rfloor, \\
\mu_{i, j}(n) & =\sum_{k=1-p}^{p-1} C_{i}(k) C_{j}(k+n),
\end{aligned}
$$

$C_{i}(k)$ and $C_{j}(k)$ in (20) are the aperiodic ACFs of codes $i$ and $j$, respectively. The parameter $\mu_{i, j}(n)$ can also be expressed using aperiodic CCFs, $C_{i, j}(k)$, between codes $i$ and $j$ :

$$
\mu_{i, j}(n)=\sum_{k=1-p}^{p-1} C_{i, j}(k) C_{i, j}(k+n) .
$$

The crucial parameter $\mu_{i, j}(0)$ can be bounded by using the Cauchy inequality as follows

$$
p^{2}-2\left\{S_{i} S_{j}\right\}^{1 / 2} \leq \mu_{i, j}(0) \leq p^{2}+2\left\{S_{i} S_{j}\right\}^{1 / 2}
$$

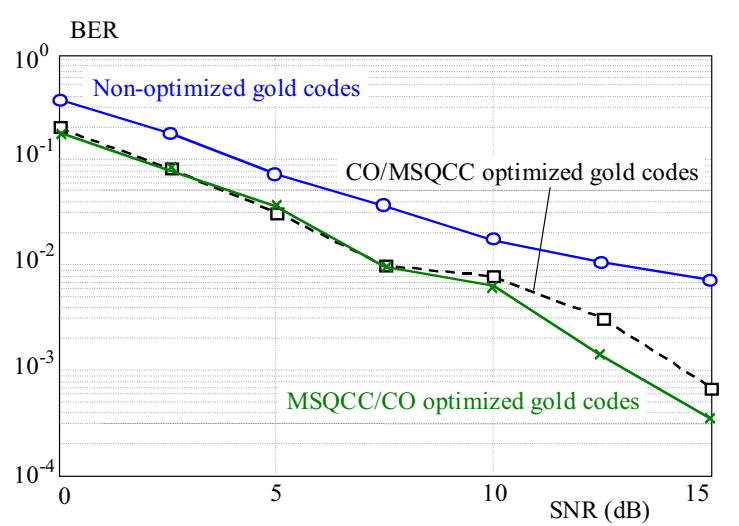

Fig. 3. BER performance of MIMO-CDMA system that used $\mathrm{CO} / \mathrm{MSQCC}$ and MSQCC/CO optimized Gold codes $(\mathrm{NFR}=0 \mathrm{~dB})$

where

$$
S_{i}=\sum_{k=1}^{p-1} C_{i}^{2}(k), \quad S_{j}=\sum_{k=1}^{p-1} C_{j}^{2}(k)
$$

are the sidelobe energies of the aperiodic ACFs of codes $i$ and $j$, respectively. Using (22), the upper bound for the AIP is defined [25]

$$
r_{i, j} \leq 4 p^{2}+6\left\{S_{i} S_{j}\right\}^{1 / 2}
$$

For available spreading codes with finite length, many researchers have studied phase optimization criteria such as LSE/AO, $\mathrm{AO} / \mathrm{LSE}, \mathrm{MSE} / \mathrm{AO}, \mathrm{CO} / \mathrm{MSQCC}$ and MSQCC/CO [14], [25-27]. All these criteria are based on the selection of codes having minimum auto-correlation sidelobes or minimum cross-correlation. In most applications where we are trying to find high performance codes, the sidelobe energy parameter may be more important than the peak correlation parameters. Therefore, it is required to determine the least sidelobe energy (LSE) phases of the PN codes. The LSE phases which have the best odd auto-correlation parameters are considered, and these phases are referred as being auto-optimal (AO) among the LSE phases. This amounts to a reversal of the arrangement in which the AO and LSE criteria are used to realize $\mathrm{AO} / \mathrm{LSE}$ phases. Hence, the notation LSE/AO for the phases which are AO among the set of LSE phases are used.

The MSE/AO proposed by Ofarrell is a powerful phase optimization criteria that may be useful to select the PN codes for using in CDMA applications [27]. The definition of the MSE/AO criteria has a similar definition of the LSE/AO criteria. The unique change is replacing of the minimization of the sidelobe energy with maximization. Although the MSE/AO rule is AO, it produces an odd auto-correlation having large sidelobes. The major object of the MSE/AO optimization criteria is to maximize the maximum absolute value of sidelobes of ACF [28]. The CO/MSQCC and MSQCC/CO are other phase optimization criteria introduced in [13]. The cross-optimal 


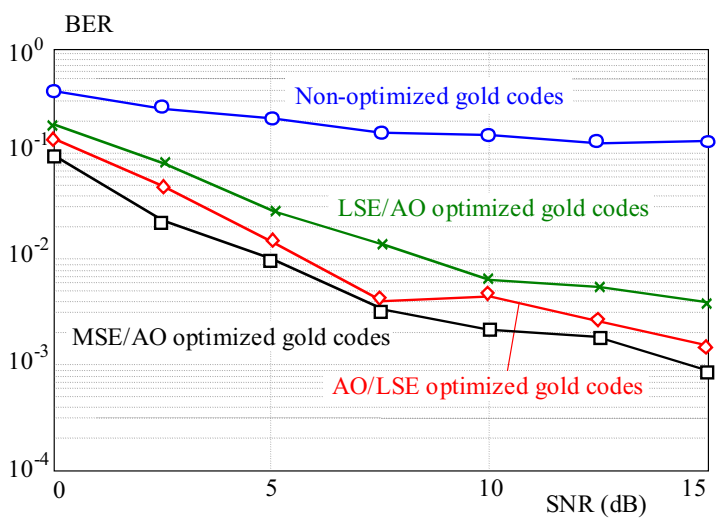

Fig. 4. BER performance of MIMO-CDMA system that used $\mathrm{LSE} / \mathrm{AO}, \mathrm{AO} / \mathrm{LSE}$ and MSE/AO optimized Gold codes (NFR=20 dB)

(CO) in these optimization criteria is defined as the sequence phase such that the peak $\mathrm{CCF}$ values occurs the least number of times. $\mathrm{CO}$ is similar to $\mathrm{AO}$ except that it uses odd periodic cross-correlation instead of odd periodic auto-correlation. The main aim of the CO/MSQCC optimization criteria is to minimize the maximum absolute value of CCF $[28,29]$. The MSQCC/CO optimization criteria can be obtained if the order of the above mentioned optimization steps are reserved.

\section{NUMERICAL RESULTS}

In this section, computer simulations are presented to show the improvement in the system performance when using initial-phase optimized PN codes instead of classical non-optimized spreading codes in both cases of nearfar ratio (NFR) of 0 and $20 \mathrm{~dB}$. The PN codes used in the simulations for signal spreading are initial-phase optimized Gold codes of length 31. These codes were optimized by using one of the criteria as LSE/AO, AO/LSE, $\mathrm{MSE} / \mathrm{AO}, \mathrm{CO} / \mathrm{MSQCC}$ and MSQCC/CO [13]. The simulations are performed for a MIMO-CDMA system where both the transmitter and the receiver is equipped with $N_{T}=N_{R}=2$ antennas. The number of active users in the system is $K=8$. The frame size is chosen as $M=4$. The BER performance of the MIMO-CDMA system is evaluated using Monte-Carlo simulation, and the number of Monte-Carlo trials was set to 3500 . We consider a quasi-static fading channel in which the channel matrix is constant over the duration of each frame.

Figures 2 and 3 show the BER performance of the MIMO-CDMA system with various initial-phase optimized Gold codes, as a function of SNR, obtained for $\mathrm{NFR}=0 \mathrm{~dB}$. In these figures, for comparison purposes with the MIMO-CDMA system that employs conventional Gold codes, we have also included the simulated performance for the non-optimized situation. These figures clearly indicate that the MIMO-CDMA system with optimized Gold codes outperforms the MIMO-CDMA system with non-optimized situation. For example, as can be seen in Fig. 2, at a BER of $10^{-2}$ the system

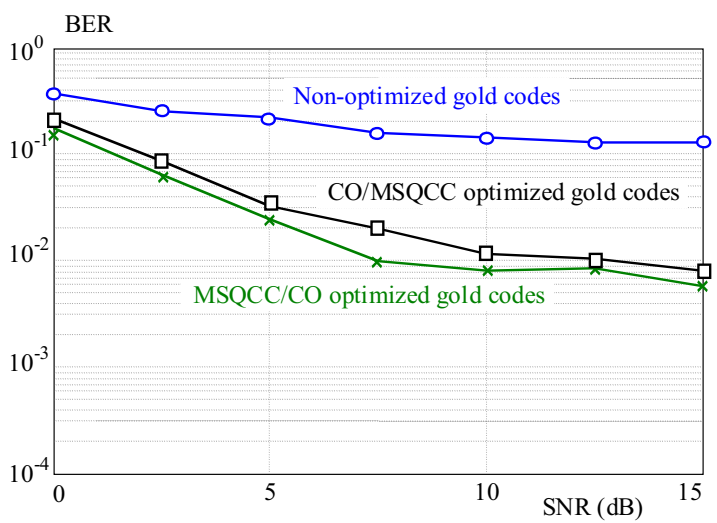

Fig. 5. BER performance of MIMO-CDMA system that used $\mathrm{CO} / \mathrm{MSQCC}$ and MSQCC/CO optimized Gold codes $(\mathrm{NFR}=20 \mathrm{~dB})$

with MSE/AO optimized Gold codes has a $7 \mathrm{~dB}$ gain over non-optimized while the system with LSE/AO and $\mathrm{AO} / \mathrm{LSE}$ optimized Gold codes has a nearly $5.5 \mathrm{~dB}$ gain. Also, Fig. 3 shows that nearly 5.5 dB gain can be achieved by using either $\mathrm{CO} / \mathrm{MSQCC}$ or MSQCC/CO optimized Gold codes at a BER of $10^{-2}$.

Figures 4 and 5 depict the improvement on BER performance of the MIMO-CDMA system when various initial-phase optimized Gold codes are employed for $\mathrm{NFR}=20 \mathrm{~dB}$. Results about using non-optimized Gold codes are also added to these figures to show the performance of employing initial-phase optimized Gold codes. It should be noted that similar findings with those observed from Figures 2 and 3 can also be seen in these figures. Again, Fig. 4 shows that the MSE/AO optimized Gold codes achieve the best performance among all the optimized codes considered. As compared with the results obtained for a low value of NFR, the effect of using initial-phase optimized Gold codes on the BER performance improvement do not decreases as the NFR value increases.

\section{CONCLUSIONS}

In this paper, we have examined the BER performance of the MIMO-CDMA system employing initial-phase optimized Gold codes in a quasi-static fading channel. Monte-Carlo simulations show that the MIMO-CDMA system with optimized Gold codes significantly outperforms the MIMO-CDMA system with non-optimized situation. It is also shown that the MIMO-CDMA system with MSE/AO optimized Gold codes gives the best performance among all the optimized codes considered in this paper. In addition, we would like to mention the following result: for high NFR case, constructive effect of initial-phase optimized Gold codes on the BER performance of the MIMO-CDMA system continues as for the low NFR condition. Therefore, it is expected that this simulative work can serve as useful contribution for communication researchers wishing to increase the BER performance of their MIMO-CDMA systems, especially in a near-far scenario. 


\section{Acknowledgements}

This research was supported by the Research Fund of Erciyes University, Grant number: FBT-07-10.

\section{REFERENCES}

[1] FOSCHINI, G. J. : Layered Space-Time Architecture for Wireless Communication in Fading Environments when Using MultiElement Antennas, Bell Labs Tech J. 1 (1996), 41-59.

[2] TELATAR, E. : Capacity of Multi-Antenna Gaussian Channels, Eur T Telecommun. 10 (1999), 585-595.

[3] WINTERS, J. : On the Capacity of Radio Communication Systems with Diversity in a Rayleigh Fading Environment, IEEE J. Sel Area Comm. 5 (1987), 871-878.

[4] ZOU, B.-DONG, Z.-LIANG, D.: Design and Performance Analysis of Orthogonal Coding Signal, Mimo-Sar, Sci China Inf Sci. 54 (2011), 1723-1737.

[5] LI, X.-JIN, S.-GAO, X.: Multi-User MIMO Downlink Eigen-Mode Transmission over Jointly Correlated MIMO Channels, Sci China Inf Sci. 54 (2011), 2124-2137.

[6] ZHA, W.-BLOSTEIN, S. D. : Improved CDMA Multiuser Receivers Robust to Timing Errors, Proceedings of IEEE International Conference on Acoustics, Speech and Signal Processing, Salt Lake City, USA, 2001, pp. 2257-2260.

[7] YOON, Y. C.-LEIB, H. : Chip-Delay Locked Matched Filter for DS-CDMA Systems using Long Sequence Spreading, IEEE T. Commun. 49 (2001), 1468-1478.

[8] Van HEESWYK, F.-FALCONER, D. D.-SHEIKH, U. H. : A Delay Independent Decorrelating Detector for Quasi-Synchronous CDMA, IEEE J. Sel. Area Comm. 14 (1996), 1619-1626.

[9] CHU, L. C.-MITRA, U.: Performance Analysis of an Improved MMSE Multiuser Receiver for Mismatched Delay Channels, IEEE T. Commun. 46 (1998), 1369-1380.

[10] CHU, L. C.-MITRA, U.: Analysis of MUSIC-Based Delay Estimators for Direct-Sequence Code-Division Multiple-Access Systems, IEEE T. Commun. 47 (1999), 133-138.

[11] ZHA, W.-BLOSTEIN, S. D.: Multiuser Receivers that are Robust to Delay Mismatch, IEEE T. Commun. 50 (2002), 2072-2081.

[12] LU, R. C. Y.: Multiuser Detection in CDMA MIMO Systems with Timing Mismatch, Dissertation for the Master of Science Degree. Ontario: Queen's University, 2004.

[13] KÄRKKÄINEN, K. H. A.-LEPPÄNEN, P. A.: The Influence of Initial-Phases of a PN Code Set on the Performance of an Asynchronous DS-CDMA System, Wir. Per. Commun. 13 (2000), 279-293, Available at: http://www.ee.oulu.fi/ kk.

[14] KRKKÄINEN, K. H. A.: Influence of Various PN Sequence Phase Optimization Criteria on the SNR Performance of an Asynchronous DS-CDMA System, Proceedings of IEEE Military Communications Conference, San Diego, CA, 1995, pp. 641-646.

[15] FOlEGOT, T.-De ROSNY, J.-PRADA, C. et al : Adaptive Instant Record Signals Applied to Detection with Time Reversal Operator Decomposition, J. Acoust. Soc. Am. 117 (2005), 3757-3765.

[16] KACHANOV, V. K.-SOKOLOV, I. V.: Features of Applying Complexly Modulated Signals in Ultrasonic Flaw Detection, Russ. J. Nondestruct. 43 (2007), 790-808.

[17] BEN JEMAA, Z.-BELGHITH, S. : Correlation Properties of Binary Sequences Generated by the Logistic Map-Application to DS-CDMA, Proceedings of IEEE International Conference on Systems, Man and Cybernetics, Hammamet, Tunisia, 2002, pp. $447-451$.

[18] YOSHIOKA, D.-TSUnedA, A.-INOUE, T. : Initial Phase Selection of Chaos-Based Spreading Sequences in Asynchronous DS-CDMA Systems, Proceedings of IEEE 8th International
Symposium on Spread Spectrum Techniques and Applications, Sydney, Australia, 2004, pp. 713-717.

19] DEVELI, I. : Detection of Optimal Spreading Codes for DSCDMA Wireless Systems with Despreading Sequences Weighted by Adjustable Chip Waveforms, J Franklin I. 342 (2005), 69-84.

[20] DEVELI, I.-CIFTLIKLI, C. : Computation of the Tuning Values of Exponential Chip Weighting Waveforms for DS-CDMA Communications over AWGN Channels, IEE P. Commun. 151 (2004), 132-136.

21] FILIZ, M.: Performance Analysis of Multiuser CDMA-MIMO Systems using Initial-Phase Optimized Pseudo Noise Code Sequences, Dissertation for the Master of Science Degree. Kayseri: Erciyes University, 2007. (in Turkish)

22] ABUHILAL, H.-HOCANIN, A.-BILGEKUL, H.: Robust MIMO-CDMA Decorrelating Detector, Proceedings of IEEE International Conference on Signal Processing and Communications, Dubai, UAE, 2007, pp. 732-735.

23] PURSLEY, M. B.: Performance Evaluation for Phase-Coded Spread-Spectrum Multiple- Access Communication - Part I: System Analysis, IEEE T. Commun. 25 (1977), 795-799.

24] PURSLEY, M. B.-SARWATE, D. V.: Performance Evaluation for Phase-Coded Spread-Spectrum Multiple-Access Communication - Part II: Code Sequence Analysis, IEEE T. Commun. 25 (1977), 800-803.

25] PURSLEY, M. B.-ROEFS, H. F. A.: Numerical Evaluation of Correlation Parameters for Optimal Phases of Binary Shift-Register Sequences, IEEE T. Commun. 27 (1979), 1597-1604.

26] SARWATE, D. V.-PURSLEY, M. B. : Crosscorrelation Properties of Pseudorandom and Related Sequences, P. IEEE 68 (1980), 593-619.

27] OfARRELL, T.: New Signature Code Sequence Design Techniques for CDMA Systems, Electron Lett. 27 (1991), 371-373.

28] ARMANAVIČIUS, G.-KAŽYS, R. : Analysis of Pseudo Noise Sequences for Multi Channel Distance Measurements, Ultragarsas 37 (2000), 16-23.

29] El-KHAMY, S. E.-GAD, M. M.-SHAABAN, S. E.: Optimization of Chaotic Spreading Codes for Asynchronous DSCDMA Applications using Genetic Algorithms, Proceedings of 22nd National Radio Science Conference, Cairo, Egypt, 2005, pp. 205-216.

Received 18 July 2012

Ibrahim Develi was born in Bielefeld, Germany on June 10,1973 . He received the BS, MS and PhD degrees in electronics engineering from Erciyes University, Turkey, in 1995, 1997 and 2003, respectively. From May 2003 to June 2009, he was an Assistant Professor with the Department of Electrical and Electronics Engineering, Erciyes University. Currently, he is an Associate Professor at the same department teaching wireless communications and his current research interests are in spread spectrum communications, multiuser communications, wireless networks, radio-over-fibre transmission, radiocommunication, millimeter waves, multiple access interference rejection in DS-CDMA systems, and applications of neural networks to multiuser communication systems. Dr. Develi is currently serving as an Associate Editor for the EURASIP Journal on Wireless Communications and Networking.

Meryem Filiz was born in Van, Turkey on May 10, 1983. She received the BS and MS degrees in Electrical and Electronics Engineering from Erciyes University, Turkey, in 2005 and 2007, respectively. Her current research interests include digital communications and MIMO-CDMA systems. She is working toward her Ph.D. in the area of pulse design for UWB communication systems. 